\title{
Capacitación en la empresa mexicana: un estudio de formación en el trabajo*
}

\author{
Training in the Mexican company: a study of job training
}

Recibido: 21 de marzo de 2014 - Revisado: 13 de agosto de 2014 - Aceptado: 06 de noviembre de 2014

\author{
Alma Lilia Sapién Aguilar** \\ Laura Cristina Piñón Howlet ${ }^{* * *}$ \\ María del Carmen Gutiérrez Díez ${ }^{* * *}$
}

\section{Resumen}

Los procesos de capacitación y adiestramiento en la mediana empresa mexicana no están siendo entendidos como una inversión que contribuya al éxito empresarial. El objetivo del artículo es analizar estos procesos. El enfoque metodológico que se utilizó es de tipo explorativo y descriptivo con un diseño no experimental o ex post facto. Se eligieron aleatoriamente 17 medianas empresas. Los resultados indican que en la mediana empresa mexicana se deben emprender acciones contundentes que sirvan para que la capacitación y el adiestramiento dejen de ser vistos como un gasto innecesario. Es menester que se tomen como la mejor inversión que pueda hacerse en recursos humanos. Por lo anterior, la capacitación y el adiestramiento deben convertirse en parte de la cultura laboral de toda organización.

\section{Palabras clave}

Capacitación, adiestramiento, mediana empresa, cultura laboral.

\begin{abstract}
The processes of training and instruction in a medium-sized Mexican company are not being understood as an investment that contributes to business success. The aim of this study was to analyze these processes. The methodological approach was exploratory and descriptive with a non-experimental or ex post facto design. The Mexican Business Information System was consulted and 17 medium-sized companies in the industrial sector were randomly chosen. The results indicated that in the median Mexican company decisive actions must be taken in order to achieve that training and instruction are no longer seen as an unnecessary expense. They need to be understood as the best investment one can make in human resources. Therefore, training and instruction must become a part of the work culture of any organization.
\end{abstract}

\section{Keywords}

Training, instruction, medium-sized companies, wwork culture.

\footnotetext{
* Artículo de investigación. Proyecto de investigación sobre la capacitación en las medianas empresas en la ciudad de Chihuahua, estado de Chihuahua, México.

** Doctora en Administración por la Universidad Autónoma de Chihuahua; profesora de tiempo completo desde 1993 a la fecha. Autora de varios libros y artículos de investigación y docencia.. Docente investigadora de la Facultad de Contaduría y Administración de la Universidad Autónoma de Chihuahua, Chihuahua, México. Correo electrónico:

lsapien@uach.mx

**** Doctora en Administración por la Universidad Autónoma de Chihuahua; catedrática de tiempo completo desde 1993 a la fecha. Autora de libros y artículos de investigación y docencia. Ha presentado trabajos de investigación en congresos nacionales e internacionales. Sus líneas de investigación son: innovación tecnológica, comportamiento organizacional, administración con valores y educación. Profesora investigadora de la Facultad de Contaduría y Administración de la Universidad Autónoma de Chihuahua, Chihuahua, México. Correo electrónico: lpinon@uach.mx

***** Doctora en Administración por la Universidad Autónoma de Chihuahua; catedrática de tiempo completo desde 2006 a la fecha; anteriormente se desempeñó como analista de sistemas en la industria manufacturera de exportación. Sus líneas de investigación son: innovación tecnológica, comportamiento organizacional, administración con valores y educación. Docente investigadora de la Facultad de Contaduría y Administración de la Universidad Autónoma de Chihuahua, Chihuahua, México.

Correo electrónico:

cgutierr@uach.mx
} 


\section{Introducción}

La capacitación y el adiestramiento son formas de educación. Por medio de ellas, se adquieren conocimientos, habilidades y destrezas que son necesarios para lograr la eficacia y la excelencia en la realización de tareas, funciones y responsabilidades. En específico, la capacitación es un proceso educacional de carácter estratégico, aplicado de manera organizada y sistemática que modifica actitudes (William, 1993). Por su parte, el adiestramiento proporciona conocimientos en los aspectos técnicos del trabajo (Ayala, 2004). La nueva cultura laboral busca impulsar la capacitación de trabajadores y empresarios de forma continua (Amitabh \& Manjari, 2004). Lo anterior en la búsqueda de mejorar el nivel de vida de los individuos y de optimizar la calidad de una organización, provocando de este modo, beneficios para ambos (Vázquez, 1997). Yang y Yang (2010) mencionaron que la mayoría de las empresas privadas en China presta escasa atención a los programas de capacitación y adiestramiento, lo que representa un problema común para todas las empresas.

El desarrollo debe comprender íntegramente al hombre (Rubio, 2004), es decir, debe tener en cuenta toda la formación de su personalidad: carácter, hábitos, educación de la voluntad, cultivo de la inteligencia, sensibilidad hacia los problemas humanos y capacidad para dirigir. En otras palabras, es el proceso para acentuar o adquirir valores, estilos, trabajo en equipo y otras facetas de la personalidad (Arias \& Heredia, 2010). Al usar la terminología de la lógica tradicional, si se considera el género como la educación, entonces, la especie sería la capacitación y una subespecie sería el adiestramiento. El resultado pretendido y previsto es el desarrollo psicológico, social, tecnológico y económico de los individuos así como de los grupos, de las organizaciones y de los países (Rodríguez \& Ramírez, 1991).

A pesar de la gran importancia que representan las compañías pequeñas y medianas de
México, estas difícilmente cuentan con programas actualizados y dinámicos de capacitación. $\mathrm{Y}$, en general, los programas de capacitación no han sido tampoco una demanda central de los sindicatos (Sánchez-Castañeda, 2007). Está claro que la capacitación y el adiestramiento, deben estimarse como una herramienta fundamental de las políticas públicas de un país. Lo dicho, para garantizar el ingreso al empleo, la permanencia en el mismo, o bien, la reconversión hacia otra actividad en caso de su pérdida. Además de ser un presupuesto de empleabilidad, es un instrumento económico que mejora la productividad-competitividad de las empresas (Sánchez-Castañeda, 2007).

Las actuales tendencias que determinan la conducta de las empresas se están configurando en torno a la globalización y de acuerdo con el avance tecnológico. Una parte significativa de las ventajas competitivas de la actividad económica reside en el bagaje de conocimientos, destrezas, habilidades y capacidades que posee la fuerza de trabajo (Vázquez, 1997). No obstante, en México, los programas de capacitación y desarrollo son sumamente escasos y limitados. La administración de recursos humanos tiene como una de sus tareas proporcionar la capacitación humana, requerida por las necesidades de los puestos o de la organización. Por lo anterior, el objetivo de este estudio fue analizar el proceso de capacitación y adiestramiento en 17 medianas compañías del sector industrial de la ciudad de Chihuahua, estado de Chihuahua, México, para identificar la forma en que se definen las necesidades de capacitación.

Un segundo objetivo fue establecer si el proceso de capacitación y adiestramiento es sistemático y se realiza en tiempo y circunstancias adecuadas, precisando a quiénes se dirige dicho proceso. El reconocer la relevancia de la capacitación y el desarrollo para introducir un cambio de actitudes y conductas que promuevan la creación de una cultura laboral, traerá como consecuencia un incremento en la productividad y la competitividad de los trabajadores y de las empresas. 


\section{Marco conceptual}

\section{El marco legal de la capacitación en México}

La Constitución Política de los Estados Unidos Mexicanos indica las obligaciones que el empleador tiene de surtir capacitación y adiestramiento al trabajador, ya sea dentro o fuera de su horario de trabajo, tales como: a) "La Federación, los estados y los municipios tienen la obligación de garantizar e incrementar la capacitación productiva destinada a los pueblos indígenas" (Constitución Política de los Estados Unidos Mexicanos, art. 2, apartado B, fracción II), y b) "La Federación y los estados deben proporcionar capacitación para el trabajo como un medio de readaptación social" (Constitución Política de los Estados Unidos Mexicanos, art. 18).

Así también, el empleado tiene como deber capacitarse dentro de la empresa, lo señala el artículo 123, apartado A, fracción XIII de la Constitución Política mexicana, donde expresa que: "Las empresas cualquiera que sea su actividad, estarán obligadas a proporcionar a sus trabajadores, capacitación o adiestramiento para el trabajo. La ley reglamentaria determinará los sistemas, métodos y procedimientos conforme a los cuales los patrones deberán cumplir dicha obligación".

La Ley Federal del Trabajo, hace referencia a la capacitación y el adiestramiento que dispone la Carta Política en el capítulo III bis: "Todo trabajador tiene el derecho a que su patrón le proporcione capacitación o adiestramiento en su trabajo que le permita elevar su nivel de vida y productividad" (art. 153-A).

Toda empresa pública o privada está en la obligación de facilitar a sus trabajadores capacitación, de acuerdo con la Ley Federal del Trabajo, se debe fijar este derecho del empleado en el reglamento interno de cada empresa. Aunque no exista un contrato de trabajo clara- mente definido rige la ley en mención (AguilarMorales, 2010).

\section{Materiales y métodos}

La investigación se ubicó en el campo de las ciencias sociales dentro del tipo exploratoria, ya que el objetivo de la misma fue examinar un tema o problema de investigación poco estudiado. Además se clasifica como descriptiva porque comprende la descripción, registro, análisis e interpretación de la naturaleza actual, composiciones o procesos relacionados con la capacitación y adiestramiento de la mediana empresa en México (Sampieri, Fernández, \& Baptista, 2003). Según el control sobre las variables, este estudio investigativo corresponde con el talante de un diseño del tipo no experimental o ex post facto, puesto que se observa la situación o el fenómeno tal como se da en un contexto natural, para después examinarlo. Por otra parte, se miden independientemente los conceptos o variables que tienen que ver con la capacitación y adiestramiento en las empresas del área de análisis.

El universo del presente estudio comprendió los directores o gerentes de las medianas empresas en la ciudad de Chihuahua, estado de Chihuahua, México en el periodo entre junio de 2010 y agosto de 2012. Para la realización de esta investigación, en una primera etapa se consultó la página del Sistema de Información Empresarial Mexicano (SIEM), de donde se obtuvo una relación de 38 medianas empresas del sector industrial que se encuentran en la ciudad de Chihuahua. Estas firmas cuentan con un número de empleados que va de 51 hasta 250. De este universo de medianas empresas (38), para calcular el tamaño de la muestra se utilizó la fórmula para poblaciones finitas. Con la aplicación de esta ecuación fue posible estimar el tamaño de muestra requerido para garantizar la normalidad estadística, dando una muestra de 17 compañías, las cuales se dedican a los servicios profesionales y administrativos, a la construcción en general, a la construcción de carreteras, puentes y similares, a 
la elaboración de concreto, al procesamiento de harina de maíz, a la pasteurización, envasado y distribución de lácteos, a la compraventa y servicios de equipo de comunicación, a la elaboración de café, a la manufactura y acabados de troqueles metálicos, al giro metalmecánico, a la construcción de vías terrestres, a la venta y distribución de gas licuado del petróleo, a la comercialización de carnes, a la fabricación de puertas y molduras y a la compraventa e industrialización de productos cárnicos.

Para recolectar los datos se usó como instrumento un cuestionario estructurado, previamente validado por expertos. El primer contacto con cada empresa se llevó a cabo vía telefónica, con el propósito de dar información del proyecto y solicitar la colaboración. Una vez se obtuvo la aceptación para participar en el estudio, se les mandó el cuestionario que constaba de un total de 20 preguntas. Las preguntas 1, 2, $3,4,10$ y 15 eran de tipo binomial, mientras que el resto contenían respuestas de opción múlti- ple. El instrumento se envió a través de correo electrónico. Antes de presentar los interrogantes se solicitó información adicional sobre fecha, empresa, nombre, puesto, correo electrónico y teléfono. Al término de la aplicación de los cuestionarios, se procedió a elaborar una base de datos para luego graficarlos y analizarlos utilizando estadística descriptiva.

\section{Resultados}

Primera pregunta: ¿cuentan con un presupuesto asignado a la capacitación? $47 \%$ de las empresas no cuenta con un presupuesto designado a esta área (gráfica 1). Esto no significa que las empresas no lleven a cabo la capacitación de los empleados, sino que conforme surgen las necesidades de capacitación y van programándose los cursos, el presupuesto es requerido y luego asignado y ejecutado. Resulta importante señalar que $53 \%$ de las compañías sí tienen contemplado en el presupuesto los gastos para capacitación.

Gráfica 1.

Presupuesto, comisión mixta, planes y programas y área de capacitación

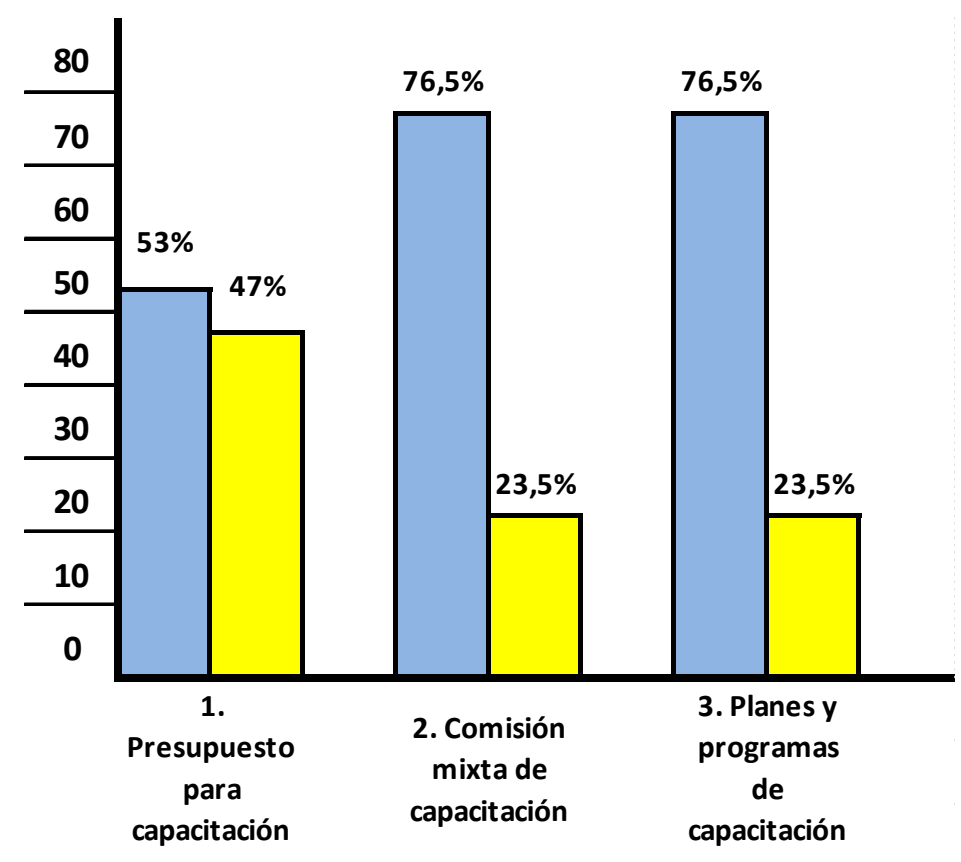

Fuente: Sapién (2012). 
Segunda pregunta: ¿existe en la empresa una comisión mixta de capacitación y adiestramiento legalmente constituida y registrada ante la Secretaría del Trabajo y Previsión Social (STPS)? En la parte central de la gráfica 1, se muestra que el 76,5\% de las empresas no cuentan con una comisión mixta de capacitación y adiestramiento legalmente constituida y registrada ante la STPS. El 23,5 \% restante se consideró alto, en virtud de que representa casi la cuarta parte de las empresas entrevistadas.

Tercera pregunta: ¿cuentan con planes y programas de capacitación y adiestramiento debidamente registrados ante la STPS? Las úl- timas dos barras de la gráfica 1 revelan que el $76,5 \%$ de las empresas cuentan con dichos planes, mientras que el $23,5 \%$ restante no, porcentaje que se valoró como alto, en virtud de que representa casi la cuarta parte de las empresas entrevistadas.

Cuarta pregunta: ¿en esta empresa, se dispone de un área responsable de la capacitación?, $53 \%$ de las firmas cuentan con un área concreta responsable de capacitación y $47 \%$ no tiene un área particular (gráfica 2), lo anterior posiblemente se debió a que dado el tamaño de las compañías, no existe un nivel de departamentalización específico.

Gráfica 2.

Área y niveles de capacitación

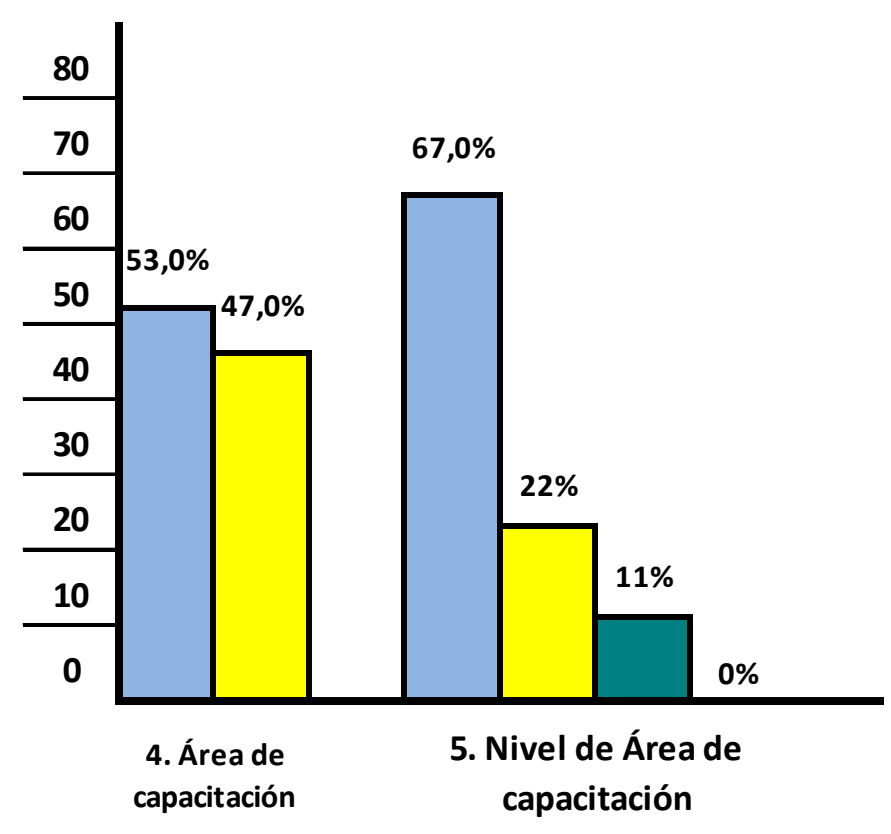

Fuente: Sapién (2012).

Quinta pregunta: ¿a qué nivel se ubica el área de capacitación en esta empresa? Las tres últimas barras de la gráfica 2 muestran que en el $22 \%$ de las empresas entrevistadas se encuentra a nivel de gerencia; en el $67 \%$ a nivel jefatura de departamento y el $11 \%$ de las empresas no lo precisó.
En el número seis del cuestionario se realizó el siguiente planteamiento: ¿los formatos que exige la STPS son: fáciles de llenar, difíciles de llenar o se desconocen los formatos? La gráfica 3 evidencia que en el 23,5\% de las empresas entrevistadas se desconocen dichos formatos. Este porcentaje es alto, ya que esto 
significó que en esas firmas nunca se ha impartido una capacitación en forma sistemática.

El interrogante siete preguntó si la legislación en materia de capacitación y adiestramiento era: fácil de cumplir, difícil de cumplir o si se desconocía en detalle la legislación en la materia. Este resultado se presenta en la parte central de la gráfica 3 , donde es obvio que en promedio el $32 \%$ de las compañías desconoce en detalle la legislación en materia de capacitación y adiestramiento. Este porcentaje se valoró como alto, dada la importancia que tiene, principalmente para los responsables del área de recursos humanos, estar actualizados y bien instruidos en dicho tema.

Gráfica 3.

Formatos y legislación en capacitación

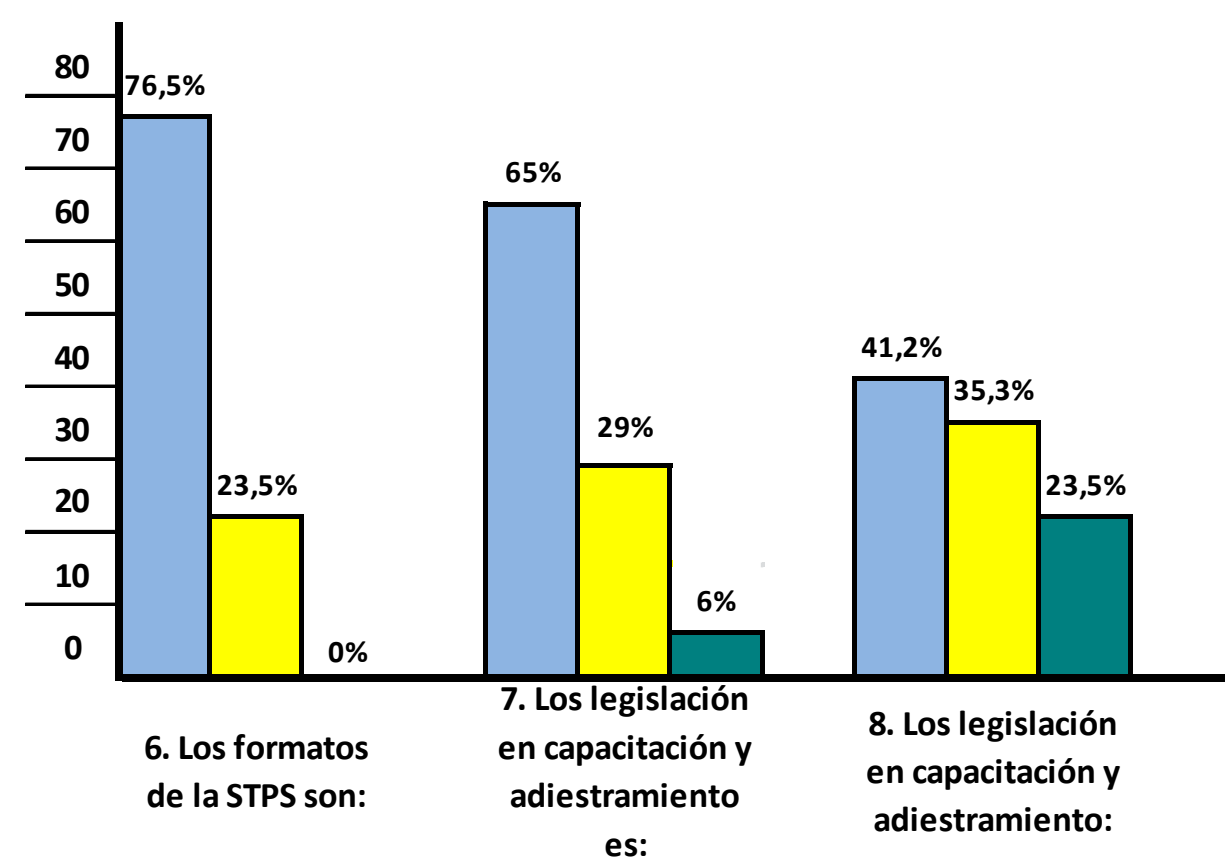

Fuente: Sapién (2012).

En la pregunta ocho se planteó si la legislación sobre capacitación y adiestramiento se ajustaba a la realidad del país, necesitaba modificarse o si se desconocía. Las tres últimas barras de la gráfica 3 muestran que el 41,2\% de las empresas entrevistadas opinó que se ajusta a la realidad; el $23,5 \%$ que necesita modificarse y el $35,5 \%$ que desconoce la legislación en esta materia. Se consideró este último resultado sumamente alarmante, puesto que representa más de una tercera parte de las firmas entrevistadas.

Novena pregunta: ¿cómo considera las actividades de capacitación? Se dieron las si- guientes opciones de respuesta: como generadoras de incrementos en la productividad; como generadoras de incrementos en la competitividad; solo para dar cumplimiento a las obligaciones legales, que de no ejecutarse generarían el pago de multas; o como un medio para favorecer el crecimiento del personal y para evitar la obsolescencia de la organización.

En la gráfica 4 se observa que estos resultados fueron muy positivos, ya que el $53 \%$ las consideró generadoras de aumentos en la productividad; $59 \%$ como generadoras de incrementos en la competitividad y el $12 \%$ solo para dar cumpli- 
miento a las obligaciones legales que de no llevarse a cabo ocasionarían el pago de multas. Las empresas que repararon en que la capacitación es un medio para favorecer el crecimiento del personal y para evitar la obsolescencia de la organización sumaron un $65 \%$, y solo el $6 \%$ de las empresas entrevistadas, nunca se ha capacitado.
Décima pregunta: ¿antes de iniciar las actividades de capacitación, se realiza un programa de detección de necesidades? Las empresas que detectan necesidades antes de iniciar las actividades de capacitación representan la gran mayoría, con un 76,5 \% (gráfica 4).

Gráfica 4.

Incidencia de la capacitación y detección de necesidades

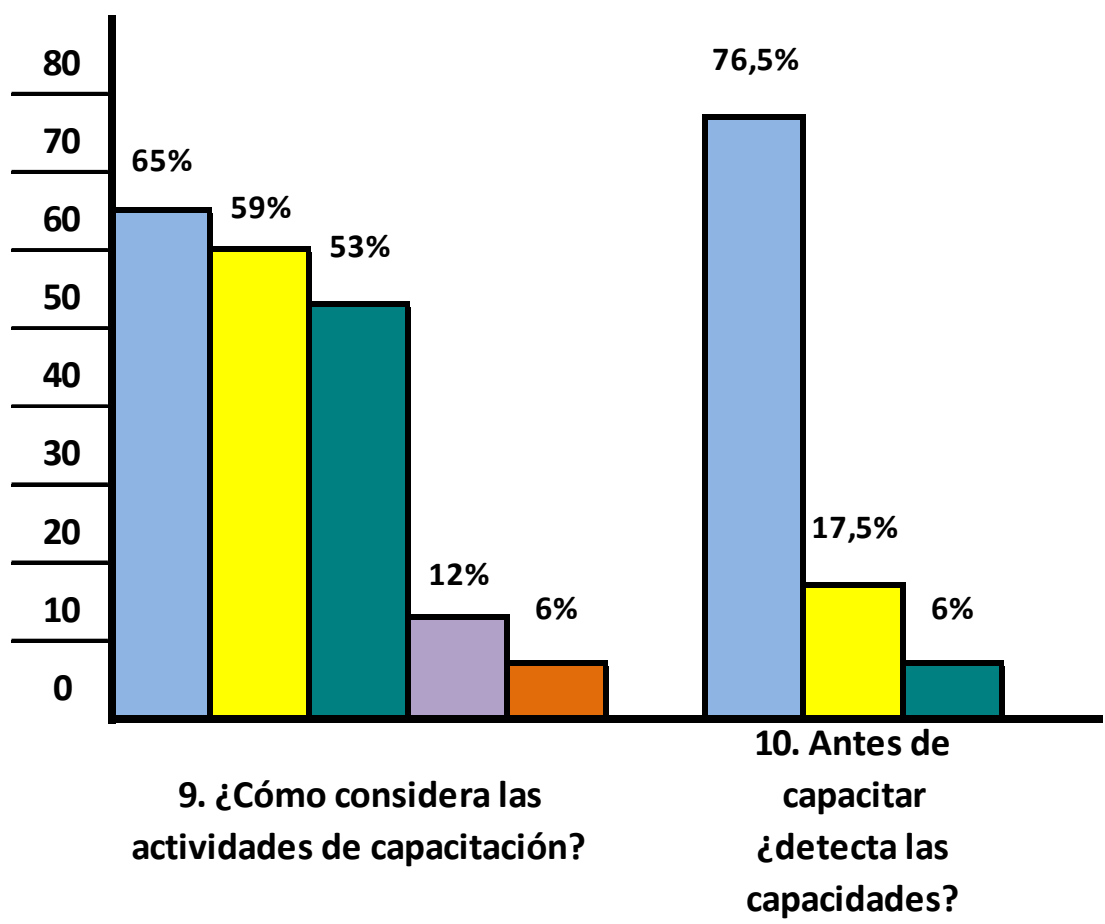

Fuente: Sapién (2012).

En el número once se realizó el siguiente planteamiento: la detección de necesidades de capacitación se realiza a través de: una entrevista con el jefe inmediato respectivo; un análisis de puestos; un análisis de los problemas más frecuentes; estudios de productividad; estudios de competitividad. La gráfica 5 señala que es por medio de los análisis de puestos con el $53 \%$ y del análisis de los problemas más frecuentes con el $59 \%$, como se planean sus planes y programas; mediante estudios de productividad $\mathrm{y}$ de estudios de competitividad solo un 12 $\%$, en ambos casos. Lo anterior indicó, que el contenido de esos planes y programas responde a actividades u oficios clásicos.

La pregunta doce en el cuestionario fue: ¿cuáles de estas actividades se realizan en la empresa en materia de capacitación? Se presentaron las siguientes opciones: se establecen objetivos conductuales a lograr mediante la capacitación; se elaboran registros de personal capacitado indicando el nivel de conocimiento y habilidades de cada trabajador; se ejecutan evaluaciones antes y después de la capacitación; se hace seguimiento de la capa- 
citación; se registran índices de costo-beneficio de la capacitación. Las últimas seis barras de la gráfica 5 revelan que el $70,5 \%$ de las empresas lleva un registro del personal capacitado; el $59 \%$ evalúa antes y después de la capacitación y el $53 \%$ efectúa un seguimiento de la capacitación. No obstante, dos de las actividades más importantes tuvieron muy bajos porcentajes: el establecer objetivos conduc- tuales, es decir, solo el 35,3\% consideró que una de las finalidades de la capacitación es el de modificar conductas; y la de llevar a cabo un registro de índices de costo-beneficio de la capacitación, que se realiza solo en un $12 \%$ de las empresas entrevistadas. Cabe mencionar que esta es una de las principales razones por las cuales las empresas no hacen capacitación sistemática.

Gráfica 5.

Detección de necesidades y actividades de capacitación

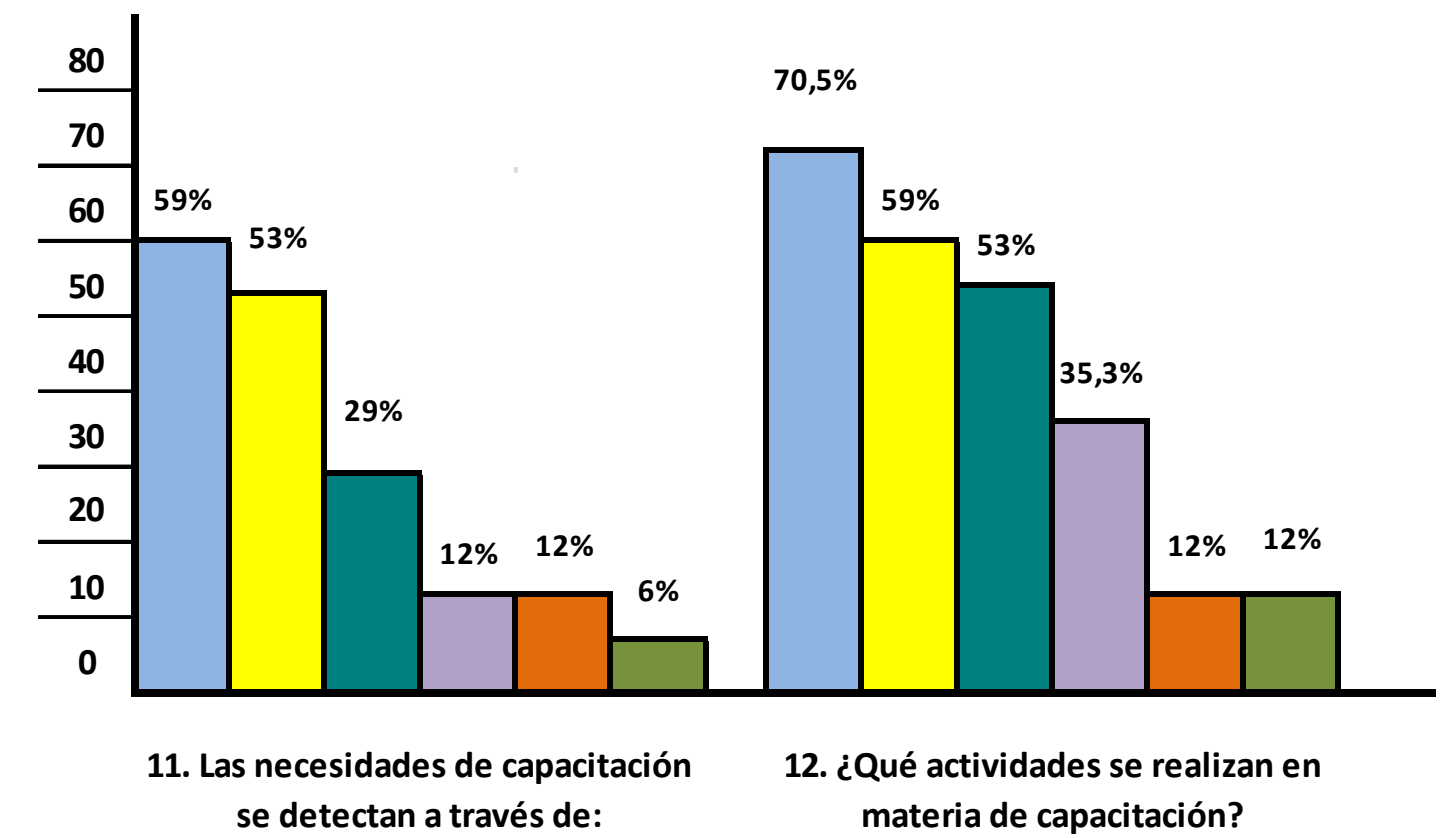

$70,5 \%$

11. Las necesidades de capacitación se detectan a través de:

\section{2. ¿Qué actividades se realizan en materia de capacitación?}

Fuente: Sapién (2012).

Pregunta trece: ¿quiénes elaboran los planes de capacitación?, dándose dos opciones: el área responsable y el área involucrada o la comisiónmixtadecapacitación yadiestramiento. La gráfica 6 muestra al área responsable y al área involucrada con un resultado del 70,5\%, seguido del personal que integra la comisión mixta de capacitación y adiestramiento con un $23,5 \%$. Puede decirse que estos resultados fueron buenos, significando que los cursos que se imparten son los adecuados, aunque lo anterior no implicó que la capacitación sea integral.

Pregunta catorce: ¿qué tipo de instructores utiliza con mayor frecuencia, interno o externo? El resultado que se obtuvo fue que se emplean instructores internos e instructores externos en la misma proporción, según se constata en la parte central de la gráfica 6 . 
Gráfica 6.

Planes, instructores y aulas de capacitación

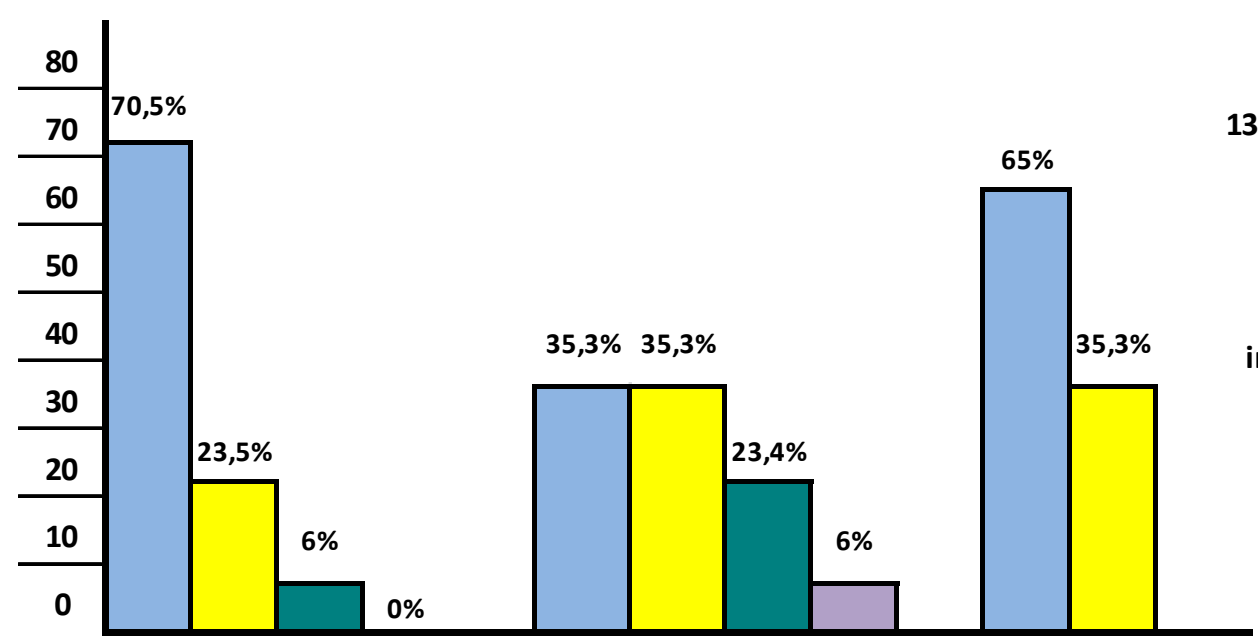

13. ¿Quiénes eleboran

los planes de

capacitación?

14. ¿Qué tipo de instrictores utiliza?

15. ¿Cuenta con aula de capacitación?

Fuente: Sapién (2012).

Pregunta quince: ¿cuenta en la empresa con aulas propias para la capacitación? El hecho de que el $65 \%$ de las compañías cuente con aulas propias para impartir su capacitación, denotó una buena intención. Esto conlleva importantes beneficios como un ahorro en gastos de capacitación y evitar que el personal se desplace fuera de la empresa, lo cual implica, entre otras cosas, un ahorro más en tiempo (gráfica 6).
Pregunta dieciséis: ¿cuál es la actitud del personal en cuanto a la capacitación: muy interesados, razonablemente interesados o algo interesados? Los resultados no se observaron muy positivos, ya que se consideró que el personal muy interesado debería estar al menos, por encima del $50 \%$. En donde menos se conoce la actitud del personal, con un resultado del $6 \%$, es porque nunca se ha impartido capacitación (gráfica 7).

Gráfica 7.

Actitud del personal frente a la capacitación y capacitación por tipo de empleo

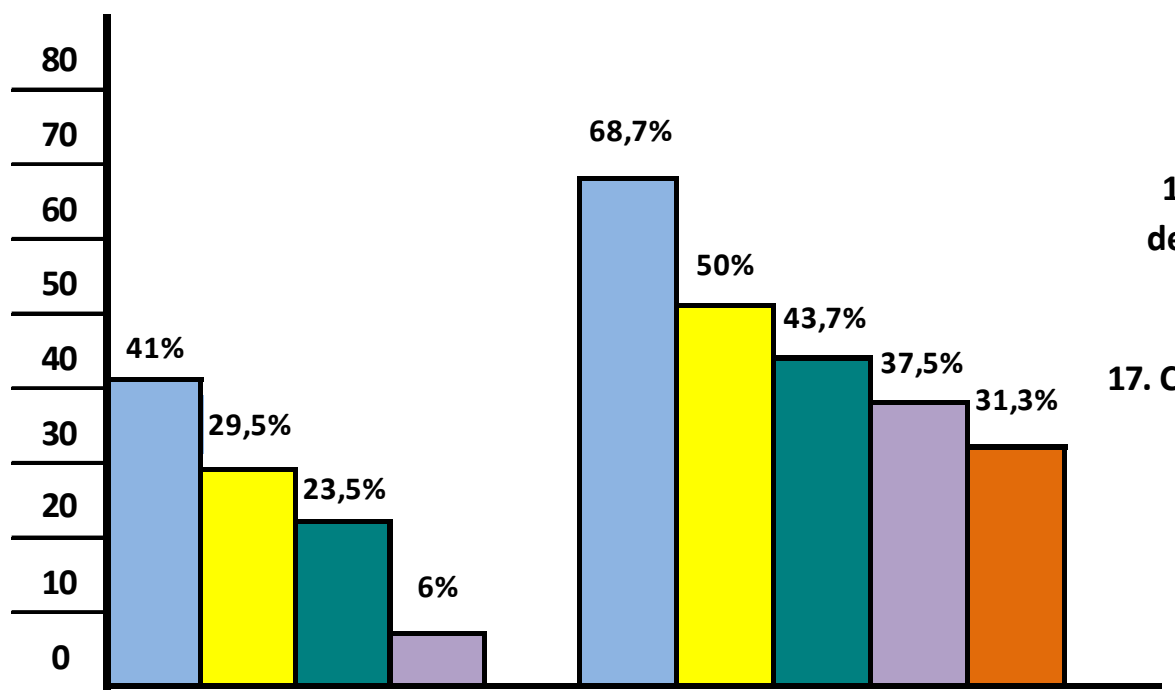

16. ¿Cuál es la actitud del personal frente a la capacitación?

17. Capacitación por tipo de empleo

Fuente: Sapién (2012). 
En el número 17 del cuestionario se pidió que se indicara en forma descendente, iniciando con el número cinco, a qué tipo de empleo se le proporciona mayor capacitación, hasta llegar al número uno, que es el tipo de empleo al que se le brinda menos capacitación: obreros, empleados de oficina, supervisores, gerentes medios y ejecutivos. La gráfica 7 muestra que el 68,75\% de las empresas entrevistadas respondieron que al nivel obrero es a quien se le otorga mayor capacitación; el $31,25 \%$ y el $43,75 \%$ de las empresas respondieron que es a los empleados de oficina y a los supervisores, respectivamente, a los que se les otorga mayor capacitación después que a los obreros; el 37,5\% de las compañías dirige la capacitación a los gerentes medios, después de darles prioridad a los tres niveles antes mencionados y, finalmente, el $50 \%$ de las firmas respondieron que son los niveles ejecutivos los que menos cursos de capacitación reciben.

Pregunta dieciocho: ¿cuáles son los principales problemas enfrentados en esta empresa en la tarea de capacitar al personal? Se dieron las siguientes alternativas: falta de interés del personal; presiones de productividad que impiden de- dicarle tiempo, se ve a la capacitación como una interferencia en las labores cotidianas; no se ofrecen suficientes oportunidades de mejoramiento para el personal ya capacitado; los cursos muchas veces no se ajustan a las necesidades reales de la empresa; carencia de capacitadores efectivos; falta de adecuación de los cursos al entorno mexicano; los trámites que tienen que realizarse ante las autoridades representan la inversión de muchas horas-hombre; dificultades para el llenado de los formatos; y reducción del presupuesto asignado a la capacitación. La gráfica 8 muestra que la ausencia de interés por parte del personal como uno de los principales problemas en la tarea de capacitar, coincidió con el problema de escasez de oportunidades de mejoramiento para el personal una vez capacitado, ambos con un $28 \%$; las presiones de productividad $65 \%$ y el ver a la capacitación como una interferencia en las labores cotidianas $47 \%$, representan los más altos porcentajes, lo cual resultó paradójico. El porcentaje que correspondió a la reducción del presupuesto asignado a la capacitación es de 35 sobre cien, lo que demuestra que esta es una de las primeras actividades afectadas con la disminución de recursos.

\section{Gráfica 8.}

Principales problemas y beneficios en la tarea de capacitar

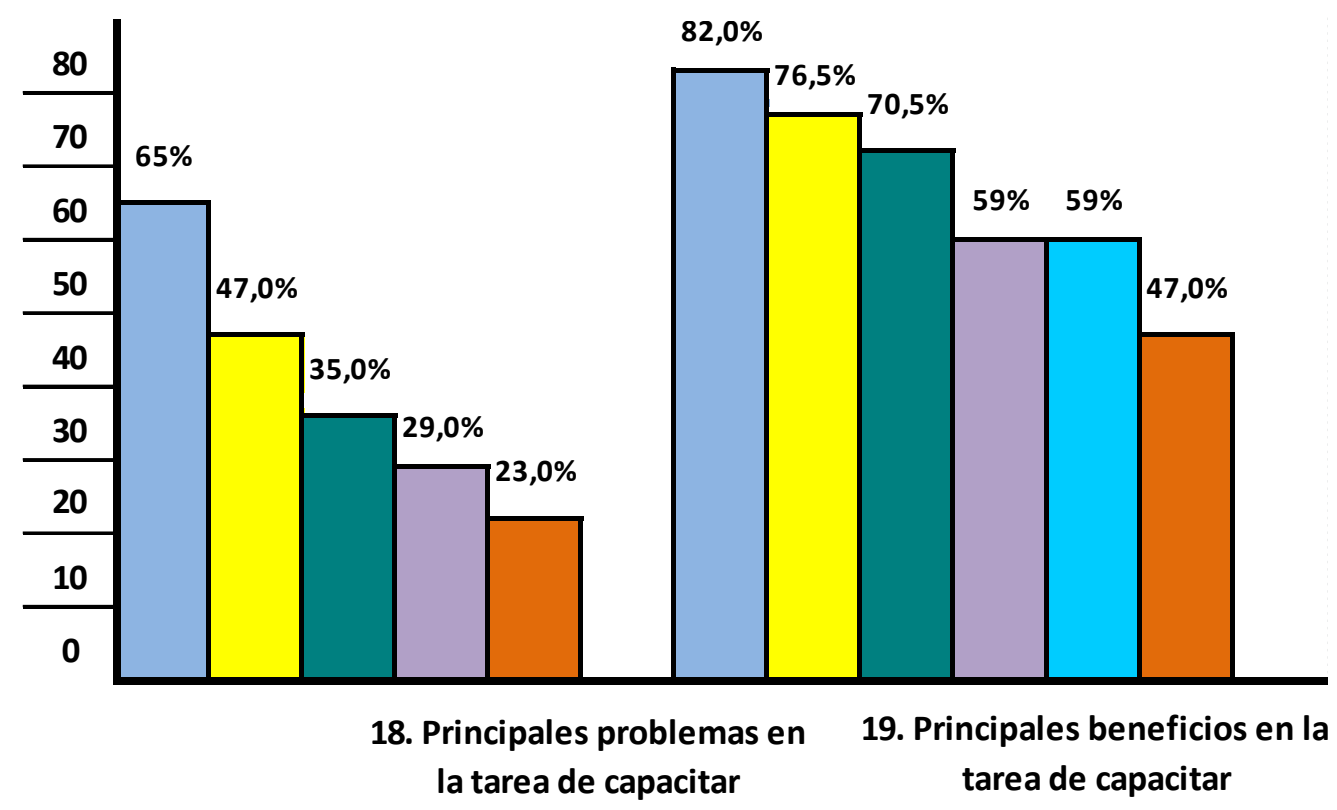

Fuente: Sapién (2012). 
Pregunta diecinueve: ¿cuáles han sido los principales logros o beneficios obtenidos en la tarea de capacitar al personal? Se presentaron las siguientes opciones: crecimiento del personal; aumento en la productividad; mayor competitividad; mejoramiento del clima de trabajo; actitud de colaboración generada; y disminución de riesgos laborales. Los principales logros o beneficios alcanzados en la tarea de capacitar, parecieron ser los ideales más que los reales. La disminución de riesgos de trabajo $82 \%$, se debió a que las empresas pertenecen al sector industrial, en donde los riesgos de trabajo implican altos costos; por lo tanto, es hacia donde está siendo enfocada la capacitación. El porcentaje que se refiere al aumento de la productividad estuvo estrechamente relacionado con la actitud de colaboración generada, ambos con el $59 \%$; y por último, la consecución de una mayor competitividad con el $47 \%$ (gráfica 8 ).

La pregunta 20 planteada en el cuestionario fue: ¿cuál considera que es el factor determinante para la competitividad de la empresa: el capital, el tecnológico o el humano? Aunque se esperaba un porcentaje más alto, fue alentador saber que la mayoría de las empresas entrevistadas considera al factor humano como el determinante para la competitividad de la empresa, con un $70,5 \%$; el otro $23,5 \%$ no pudo deslindarlo del tecnológico y solo el $6 \%$ respondió que además del factor humano y del tecnológico es igualmente importante el capital.

\section{Conclusión}

Con base en los resultados obtenidos en este estudio, puede decirse que si bien es cierto, existen empresas que realizan esfuerzos importantes por capacitar a los empleados, aún falta mucho por hacer. Todavía no hay conciencia de la relevancia que tiene el hecho de que las organizaciones cuenten con planes y programas de capacitación sistemática acordes con las exigencias de estos tiempos de constantes cambios. Se requiere también que los ejecutivos que ocupan las áreas de recursos humanos consigan convencer a los directivos e incluso al resto de los empleados, que es a través de la educación como una organización avanza, como una sociedad crece y como un país se desarrolla.

La utilización efectiva de los recursos humanos de una organización depende de la correcta aplicación de las fases de capacitación y adiestramiento, tales como: detectar necesidades de capacitación, identificar los recursos para la capacitación y adiestramiento, diseño del plan de capacitación, ejecución del programa de capacitación, la evaluación, control y seguimiento del mismo.

Todas las empresas deberían contar con una comisión mixta de capacitación y adiestramiento, así como de los planes y programas debidamente registrados ante la STPS y asignar un presupuesto anual único y exclusivo para el renglón de capacitación y adiestramiento.

\section{Referencias}

Aguilar-Morales, J. (2010) El marco legal de la capacitación en México. México: Asociación Oaxaqueña de Psicología A.C.

Amitabh, K., \& Manjari, S. (2004). Towards Effective Training and Development in Indian Public Sector Enterprises: A Casebased Analysis. South Asian Journal of Management. Recuperado de http://www. highbeam.com/doc/1P3-821274681.html

Arias, F., \& Heredia, V. (2010). Administración de Recursos Humanos para el alto desempeño. México: Trillas.

Ayala, S. (2004). Administración de Recursos Humanos. Perú: Editorial Universidad Nacional de San Martín.

Estados Unidos Mexicanos. Constitución Política de los Estados Unidos Mexicanos (1917). Congreso constituyente. Estados 
Unidos Mexicanos. Recuperado de http:// www.diputados.gob.mx/LeyesBiblio/ $\mathrm{htm} / 1 . \mathrm{htm}$

Rodríguez, M., \& Ramírez, P. (1991). Administración de la Capacitación, serie Capacitación efectiva. México: McGraw Hill.

Rubio, A. (2004). Filosofía y Ciencia; desarrollo tecnológico. Chihuahua: México.

Sánchez-Castañeda, A. (2007). La Capacitación y el adiestramiento en México, regulación, realidad y retos. Revista Latinoamericana de Derecho Social, (5), 191-228. Recuperado de http://www.rad.unam.mx

Sampieri, R., Fernandez, C., \& Baptista, P. (2003). Metodología de la investigación (3a Ed.). México: Editorial Mc Graw Hill; Interamericana Editores.

Vázquez, E. (1997). Propuesta de instauración de un sistema de capacitación al interior de la empresa en México. Revista Gestión y Estrategia, 11-12, 157-164. Recuperado de http://www.azc.uam.mx/publicaciones/gestion/num11y12/doc14.htm

William, B. (1993). Enterprise support systems: training and aiding people to plan and manage. Industrial Management. Recuperado de http://www.highbeam.com/ doc/1G1-14858457.html

Yang, H., \& Yang, X. (2010). Research on enhancing the effectiveness of staff-training in private enterprise. iBusiness, 2, 92-97. doi: 10.4236/ib.2010.21011 